\title{
Pale Kidney Sign- A Useful Ancillary Sign for Obstructive Ureterolithiasis?
}

\author{
Prafulla Kumar Dash ${ }^{1}$, Sanket² G. Satpathy³, N. R. Mohanty ${ }^{4}$

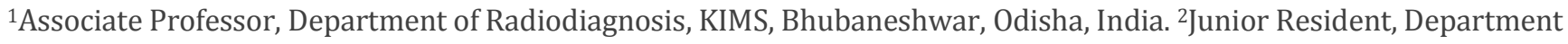 \\ of Radiodiagnosis, SCBMCH, Cuttack, Odisha, India. ${ }^{3}$ Associate Professor, Department of Radiodiagnosis, KIMS, \\ Bhubaneshwar, Odisha, India. ${ }^{4}$ Asssitant Professor, Department of Radiodiagnosis, KIMS, Bhubaneshwar, Odisha, India.
}

\section{ABSTRACT}

\section{BACKGROUND}

This study was performed to investigate the sensitivity of reduced attenuation of unilateral kidney, dubbed the 'pale kidney sign', in identification of obstructive urolithiasis.

\section{METHODS}

In this observational study, 25 patients with symptoms of acute renal colic with unilateral ureteric calculus on CT were included. Attenuation value of each kidney was averaged at 3 poles and attenuation difference between the 2 kidneys was calculated. Cut off of 5 HU (Hounsfield Units) attenuation difference in the obstructed kidney denoted pale kidney sign. Sensitivity was determined using contingency table analysis.

\section{RESULTS}

Among the 25 patients with ureteric calculus, the mean attenuation in obstructed kidney was 27.9 HU (SD 2.7) and mean attenuation in non-obstructed kidney was 33.3 HU (SD 1.9). The difference in attenuation of the obstructed and nonobstructed kidney was on an average $5.3 \mathrm{HU}$ (SD 2.6), with only 2 obstructed kidneys having higher attenuation than contralateral kidneys. Considering the cutoff of $5 \mathrm{HU}, 17$ of 25 patients had pale kidney sign with sensitivity of pale kidney sign for diagnosis of obstructing ureterolithiasis being 68\% (95\% CI- $46.5 \%$ to $88.0 \%$ ).

\section{CONCLUSIONS}

The pale kidney sign is a useful ancillary sign of obstructive ureterolithiasis with good sensitivity which can potentially be helpful in difficult cases such as those due to technical factors, confusing phlebolith, passed calculus, etc. This can reduce the number of repeat scans and avoid additional patient workup.

\section{KEY WORDS}

Pale Kidney Sign, Decreased Renal Attenuation, Ancillary Sign, Ureteric Calculus
Corresponding Author:

Sanket,

Junior Resident,

Department of Radiodiagnosis,

SCBMCH, Cuttack, Odisha, India.

E-mail:dr.sanketkulkarni@gmail.com

DOI: 10.14260/jemds/2019/608

Financial or Other Competing Interests: None.

How to Cite This Article:

Dash PK, Sanket, Satpathy G, et al. Pale kidney sign- a useful ancillary sign for obstructive ureterolithiasis? J. Evolution Med. Dent. Sci. 2019;8(36):

2803-2806,

DOI:

$10.14260 /$ jemds/2019/608

Submission 15-03-2019,

Peer Review 24-08-2019,

Acceptance 30-08-2019,

Published 09-09-2019. 


\section{BACKGROUND}

Acute flank pain is a common presenting complaint in the emergency department with ureteric colic due to ureterolithiasis being typical clinical diagnosis. Unenhanced Computed Tomography (CT) has been shown to have $>95 \%$ sensitivity, specificity and accuracy in the diagnosis of ureteric calculus.1,2 CT in many situations is used as direct visualisation of ureteric calculus on CT is considered diagnostic. However, certain masquerades like phlebolith can cause difficulty in diagnosis. ${ }^{3}$ Technical and patient factors like motion artefact and volume averaging can also cause difficulty in diagnosis of the same. Another important factor is recent spontaneous passage of the calculus which can result is additional investigation for evaluation of cause of acute flank pain, which increases the patient anxiety and healthcare load. ${ }^{4}$ To overcome these challenges many secondary CT features of renal calculus disease have been proposed like dilation of collecting system, renal enlargement, periureteric and perinephric stranding, and pale kidney sign.5,6 The Pale Kidney Sign refers to the decrease in attenuation of the obstructed kidney in comparison to non-obstructed one. It reflects lymphatic obstruction and renal oedema. This can be determined either by visual inspection (subjective method) or by measurement of attenuation values in Hounsfield Units (objective method).

The purpose of this study is to determine the sensitivity of the pale kidney sign using attenuation measurement technique in diagnosis of obstructive ureterolithiasis.

\section{METHODS}

\section{Study Population}

The study is a hospital based observational study done in the department of Radio-diagnosis, KIMS and SCBMCH between July and December 2018 on patients referred to the department with symptoms of acute renal colic for CT study. Patients with only unilateral ureteric calculus detected on CT were included in the study. Patients with history of pain longer than 4 weeks were excluded from the study. CT study was done using a helical CT technique using GE Brightspeed 16 Slice with $5 \mathrm{~mm}$ slice thickness and pitch a of 1 with the patient in supine position from the level of diaphragm to pubic symphysis in a single breath hold. No oral or intravenous contrast was used. The scans were studied using abdominal window settings (Window level $=50$, window width $=350$ ). Kidney with ureteric calculus with or without other ancillary signs like hydronephrosis was considered obstructed kidney. The other kidney without ureteric calculus and without ancillary signs of obstruction like hydronephrosis/perinephric stranding/periureteric stranding was considered non-obstructed kidney. Attenuation values of each kidney was determined by placing small Region of Interests (ROI) in upper pole, lower pole and inter polar regions in the posterior part. The upper and lower ROI were placed at the top-most and lower most slice showing collecting system respectively and the middle ROI was placed at the level of hilum. The ROIs placed were elliptical in shape and were approximately similar in size. Average of the three values that were obtained was the average attenuation of that kidney, as shown in figure 1 . The difference in attenuation was calculated between obstructed and non-obstructed kidney.

Microsoft Word was used to generate tables and data was entered in Excel spreadsheet to produce master chart. Sensitivity was determined using contingency table analysis.

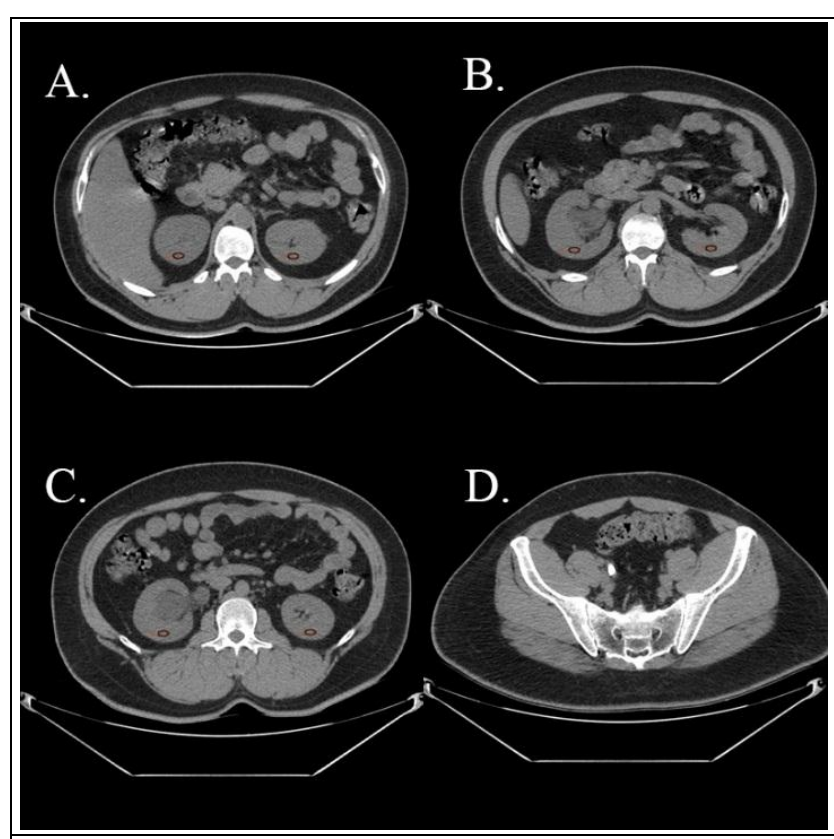

Figure 1. Showing method for determination of average attenuation of each kidney by placing ROI's. Images $A, B$ and $C$ show measurements in upper, mid and lower pole respectively. Image $D$ shows right distal ureteric calculus denoting that right side is obstructed side, consistent with patients' symptoms of acute rt. flank pain. The average attenuation of right kidney was $24 \mathrm{HU}$ and on left side was $30.6 \mathrm{HU}$, with difference in $\mathrm{HU}$ of $6.6 \mathrm{HU}$ suggestive of pale kidney.

\section{RESULTS}

The study population included 25 patients with ureteric calculus, directly visualised on CT who met the inclusion criteria. The mean attenuation of non-obstructed kidney was 33.3 Hounsfield Units (HU), with a Standard Deviation (SD) of 1.9 and range 29.8 to 38.5 . The mean attenuation in obstructed kidney was $27.9 \mathrm{HU}$, with SD of 2.7 and range 21.9 to 33.6. These findings are summarised in Table 1. The average difference in attenuation of obstructed kidney was $5.3 \mathrm{HU}$ less than non-obstructed kidney. With SD of 2.6 and range of -2.2 to 8.5. On considering cut-off value of $5 \mathrm{HU}$ for diagnosis of obstruction 17 patients out of 25 had an attenuation difference of $5 \mathrm{HU}$ or more in obstructed kidney, indicating a sensitivity of $68 \%$ (95\% Confidence Intervals $=$ $46.5 \%$ to $88.0 \%$ ). Only 2 patients had an attenuation in obstructed kidney higher than non-obstructed kidney.

\begin{tabular}{|c|c|c|c|}
\hline & $\begin{array}{c}\text { Non-Obstructed } \\
\text { Kidney (in HU) }\end{array}$ & $\begin{array}{c}\text { Obstructed } \\
\text { Kidney (in HU) }\end{array}$ & $\begin{array}{c}\text { Average Difference in } \\
\text { Attenuation (in HU) }\end{array}$ \\
\hline Mean Attenuation & $33.332 \mathrm{HU}$ & $27.96 \mathrm{HU}$ & $5.372 \mathrm{HU}$ \\
\hline Range & $29.8 \mathrm{HU}-38.5 \mathrm{HU}$ & $21.9 \mathrm{HU}-33.6 \mathrm{HU}$ & $-2.2 \mathrm{HU}-8.5 \mathrm{HU}$ \\
\hline SD & $1.9 \mathrm{HU}$ & $2.7 \mathrm{HU}$ & $2.6 \mathrm{HU}$ \\
\hline \multicolumn{3}{|c|}{ Table 1. Distribution of Attenuation Values (in Hounsfield Units) amongst } \\
Obstructed and Non-Obstructed Kidneys
\end{tabular}




\section{DISCUSSION}

Renal calculus disease (Urolithiasis) occurs secondary to multiple risk factors like low water intake, infections, congenital malformations, metabolic disorders, drugs, etc. ${ }^{7}$ Most commonly seen in middle age patients with a slight male predominance. Most calculi present with pain; however, some may remain asymptomatic. Other common symptoms are haematuria and those of secondary infection like fever, costovertebral angle tenderness (Murphy's sign). Clinical differentials include appendicitis, diverticulitis, etc with widely different management necessitating imaging for diagnosis. ${ }^{8}$

Plain X-Ray and ultrasound are most commonly used modalities to diagnose urolithiasis. Some calculi are radiolucent (e.g. Uric acid, cystine stones) on X-Ray. ${ }^{9}$ Radio opaque calculi are seen in renal fossa or along course of ureter. Phleboliths, Gall Bladder calculi and calcified nodes may mimic renal calculi. On ultrasound the calculi appear as echogenic foci with posterior acoustic shadowing and usually show twinkle artefact on colour doppler. Intrarenal gas and renal artery calcification are mimics but are not commonly encountered. Ultrasound suffers from its poor sensitivity to small calculi, ureteric calculi, reduced resolution in obese patients and its operator dependence. Intra venous urography (IVU) is another modality but is uncommonly used due risks associated with IV contrast agents and availability of CT. Computed Tomography (CT) is now considered the investigation of choice as even radiographically lucent calculi are visualised on CT with the exception of Indinavir calculi which are not visible on CT. ${ }^{9}$ Multidetector CT (MDCT) has higher sensitivity than conventional CT and also suffers from fewer artefacts (motion, volume averaging, etc.). Recent advancement in CT technology includes dual energy CT which can be used to determine stone composition, which in turn guides management.

Certain limitations of CT like motion and volume averaging artefacts can cause difficulty in diagnosis. Motion (Either voluntary or involuntary) artefacts appear as blurring, streaking, or shading. They can be reduced by patient immobilisation, sedation and by proper instructions prior to the study. Volume averaging artefact/ partial volume artefact occurs due to encompassment of different attenuation tissues in a single voxel leading to display of average attenuation of the two tissues. This can be reduced by reducing voxel size and by using thinner slices. Generally, $5 \mathrm{~mm}$ slice thickness is considered optimum for evaluation of renal calculi. Spontaneous passage of calculi can also lead to missed diagnosis and additional investigation increasing radiation exposure, patient anxiety and healthcare costs. Another differential is a phlebolith which are calcifications in venous structures and may mimic a calculus. Presence of a radiolucent centre helps differentiate from a calculus but may not be seen on $5 \mathrm{~mm}$ slice thickness. Another helpful sign that has been suggested is a soft tissue tail known as the comet tail sign which is the thrombosed or scarred vein in which the phlebolith develops. Calculus on the other hand shows a soft tissue rim sign surrounding the calculus which represents the oedematous ureteric wall, however some phleboliths may also show this sign. Use of ancillary signs has been suggested to improve sensitivity of detection of calculi in all these scenarios.
Complication of urinary tract obstruction include reduction in renal function and secondary infection. Reduction of unilateral renal function can be determined by reduced contrast excretion or striated nephrogram on IVU and dynamic CT. Renal scintigraphy can also be used to determine split renal function. Bilateral renal function deterioration causes raised renal creatinine levels. Secondary infection is best assessed using urine culture and microscopy with imaging providing a supportive role. Infection can be that of collecting system (Pyonephrosis) or of renal parenchyma (Pyelonephritis). Pyonephrosis shows debris in collecting system on USG. On CT it shows renal pelvic wall thickening, enhancement, increased density of fluid within or fluid debris levels.

Pale kidney sign refers to the unilateral decreased attenuation of obstructed kidney compared to the nonobstructed kidney. It has been suggested that this is secondary to renal oedema, hyperaemia and increased lymphatic flow leading to interstitial oedema. Some investigators used the term Decreased Renal Attenuation (DRD) to refer to the same. Attenuation difference of 5 Hounsfield Units (HU) has been suggested by multiple studies for the diagnosis of the same. However, the reported sensitivity varied from $41 \%$ to $89 \%$ in various studies. In our study the sensitivity was found to be $68 \%$ (95\% CI $=46.5 \%$ to $88.0 \%$ ). This reported variation is possibly due to timing of imaging after onset of symptoms and degree of obstruction.

Georgiades et al., ${ }^{10}$ one of the earliest to describe the sign used visual inspection of 20 cases and 20 controls, by 10 radiologists to determine if the kidney showed reduced attenuation. They relied mainly on visual inspection to determine the pale kidney and concluded that in 95\% of patients with acute renal obstruction, the affected kidney appeared to be less dense than the unobstructed kidney on visual inspection. They recommended using the sign $n$ conjunction with hydronephrosis.

Goldman et al., ${ }^{11}$ used a HU measurement technique similar to our study to determine the pale kidney in a set of 76 cases and 66 controls and reported $61 \%$ sensitivity for the same. They used a HU measurement technique as in our current study to determine its diagnostic performance to be similar to other secondary signs with the advantage being that it is the only objective measurement-based indicator. Huang, et al.,12 studied 85 cases with ureteric calculi with 30 controls and reported a sensitivity of $40.3 \%$, considering attenuation difference of $5 \mathrm{HU}$ as cut-off. They also used cutoffs of $8 \mathrm{HU}$ and $2 \mathrm{HU}$ for the same and reported a sensitivity of $12.5 \%$ and $76.4 \%$ respectively and recommended using cut off of $2 \mathrm{HU}$ and recommended using a cut off of $2 \mathrm{HU}$ due to its higher sensitivity. The trade-off of using such a low cut off was poor specificity of $76 \%$.

Other differentials for pale kidney that has been suggested including pyelonephritis and unilateral acute renal vein thrombosis. Acute Pyelonephritis presents with fever, flank pain and has positive urine culture/microscopy. Ultrasound is poorly sensitive in demonstration of pyelonephritis and may shows areas of altered parenchymal echogenicity. On non-contrast CT, Acute Pyelonephritis shows renal enlargement, perinephric stranding (Similar to obstruction) and/ or gas (In emphysematous pyelonephritis). On contrast, wedge shaped areas of reduced attenuation involving periphery (Negative rim sign), with striated 
nephrogram in excretory phase. ${ }^{13}$ Renal vein thrombosis on the other hand occurs in the backdrop of thrombogenic states, dehydration, nephrotic syndrome, etc. On doppler ultrasound, renal vein thrombosis shows absence or reversal of diastolic arterial flow (Nonspecific) and absence of flow in renal vein if vein is visualised. Contrast enhanced CT in venous phase allows visualisation of thrombus as a filling defect. ${ }^{14}$

Other ancillary signs ${ }^{5}$ that have been described include renal enlargement which can be due to dilation of collecting system or due to renal oedema secondary to same mechanism as above. Renal enlargement in isolation may be a normal variant (with left kidney being slightly larger than right), compensatory hypertrophy in contralateral renal pathologies (renal artery stenosis, renal injury, etc.), or apparent enlargement in contracted or hypoplastic contralateral kidney.

Periureteric and perinephric stranding are other ancillary signs are possibly due to lymphatic obstruction. This may also be seen in pyelonephritis. Perinephric bridging septa of Kunin suspend the kidney and may mimic perinephric stranding. Bilateral symmetric mild perinephric stranding may be normally seen in elderly population. Furthermore, this is a non-quantifiable finding. ${ }^{15}$

Dilation of collecting system (Calyces, infundibula, pelvis) known as hydronephrosis which is very useful indicator of obstructive uropathy may not always be seen in acute obstruction depending on compliance of the collecting system. A mildly obstructed system with flaccid system may show marked dilation whereas a completely obstructed system may show only minimal dilation in a low compliance collecting system. Dilatation may also be due to PUJ obstruction, strictures, malignancy, etc. Extra renal pelvis, renal sinus cysts and renal sinus varices can mimic hydronephrosis. Contrast enhanced CT can help diagnose these entities. Diuretic renogram can also be used to help differentiate extrarenal pelvis from hydronephrosis.

Limitations of our study include small population and lack of control group to determine the specificity of the sign.

\section{CONCLUSIONS}

The pale kidney sign is a useful ancillary sign of obstructive ureterolithiasis with good sensitivity which can potentially be helpful in difficult cases due to technical factors, confusing phlebolith, passed calculus, etc. This has potential to reduce repeat scans and hence radiation dose and also to potentially avoid further workup in case of passed calculus.

\section{REFERENCES}

[1] Holtz P, Begun FP, White B, et al. Ureteral calculi: diagnostic efficacy of helical CT and implications for treatment of patients. Am J Roentgenol 2013.
[2] Smith RC, Verga M, McCarthy S, et al. Diagnosis of acute flank pain: value of unenhanced helical CT. Am J Roentgenol 1996;166(1):97-101.

[3] Heneghan JP, Dalrymple NC, Verga M, et al. Soft-tissue "rim" sign in the diagnosis of ureteral calculi with use of unenhanced helical CT. Radiology 1997;202(3):709-11.

[4] Hubner WA, Irby P, Stoller ML. Natural history and current concepts for the treatment of small ureteral calculi. Eur Urol 1993;24(2):172-6.

[5] Smith RC, Verga M, Dalrymple N, et al. Acute ureteral obstruction: value of secondary signs of helical unenhanced CT. Am J Roentgenol 1996;167(5):1109-13.

[6] Boulay I, Holtz P, Foley WD, et al. Ureteral calculi: Diagnostic efficacy of helical CT and implications for treatment of patients. Am J Roentgenol 1999;172(6):1485-90.

[7] Bartoletti R, Cai T, Mondaini N, et al. Epidemiology and risk factors in urolithiasis. Urol Int 2007;79(Suppl 1):3-7.

[8] Tamm EP, Silverman PM, Shuman WP. Evaluation of the patient with flank pain and possible ureteral calculus. Radiology 2003;228(2):319-29.

[9] Saw KC, McAteer JA, Monga AG, et al. Helical CT of urinary calculi: effect of stone composition, stone size and scan collimation. Am J Roentgenol 2000;175(2):32932.

[10] Georgiades CS, Moore CJ, Smith DP. Differences of renal parenchymal attenuation for acutely obstructed and unobstructed kidneys on unenhanced helical CT: an useful secondary sign? Am J Roentgenol 2001;176(4):965-8.

[11] Goldman SM, Faintuch S, Ajzen SA, et al. Diagnostic value of attenuation measurements of the kidney on unenhanced helical CT of obstructive ureterolithiasis. Am J Roentgenol 2004;182(5):1251-4.

[12] Huang CC, Wong YC, Wang LJ, et al. Decreased renal parenchymal density on unenhanced helical computed tomography for diagnosis of ureteral stone disease in emergent patients with acute flank pain. Chang Gung Med J 2008;31(2):182-9.

[13] Johnson PT, Horton KM, Fishman EK. Optimizing detectability of renal pathology with MDCT: protocols, pearls and pitfalls. Am J Roentgenol 2010;194(4):100112.

[14] Kawashima A, Sandler CM, Ernst RD, et al. CT Evaluation of renovascular disease. Radiographics 2000;20(5):1321-40.

[15] Fukami H, Takeuchi Y, Kagaya S, et al. Perirenal fat stranding is not a powerful diagnostic tool for acute pyelonephritis. Int J Gen Med 2017;10:137-44. 